\title{
Protease Omi cleaving Hax-1 protein contributes to OGD/R-induced mitochondrial damage in neuroblastoma N2a cells and cerebral injury in MCAO mice
}

Jia-yuan WU, Mei LI, Li-juan CAO, Mei-ling SUN, Dong CHEN, Hai-gang REN, Qin XIA, Zhou-teng TAO, Zheng-hong QIN, Qing-song $\mathrm{HU}^{*}$, Guang-hui WANG*

Laboratory of Molecular Neuropathology, Jiangsu Key Laboratory of Translational Research and Therapy for Neuro-Psycho-Diseases and College of Pharmaceutical Sciences, Soochow University, Suzhou 215021, China

\begin{abstract}
Aim: In the penumbra after focal cerebral ischemia, an increase of protease Omi is linked to a decrease of Hs1-associated protein X-1 (Hax-1), a protein belonging to the Bcl-2 family. In this study we investigated the mechanisms underlying the regulation of Hax-1 by protease Omi in cerebral ischemia/reperfusion (I/R) injury.

Methods: Mouse neuroblastoma N2a cells were subjected to oxygen-glucose deprivation and reoxygenation (OGD/R); cell viability was assessed with MTT assay. Mice underwent 2-h middle cerebral artery occlusion (MCAO) and reperfusion, and the infarct volume was determined with TTC staining. The expression of Omi and Hax-1 was detected using immunoblot and immunofluorescence assays. The mitochondrial membrane potential was measured using TMRM staining.

Results: In the brains of MCAO mice, the protein level of Omi was significantly increased, while the protein level of Hax-1 was decreased. Similar changes were observed in OGD/R-treated N2a cells, but the mRNA level of Hax-1 was not changed. Furthermore, in OGD/R-treated N2a cells, knockdown of Omi significantly increased Hax-1 protein level. Immunofluorescence assay showed that Omi and Hax-1 were co-localized in mitochondria of N2a cells. OGD/R caused marked mitochondrial damage and apoptosis in N2a cells, while inhibition of Omi protease activity with UCF-101 (10 $\mu \mathrm{mol} / \mathrm{L})$ or overexpression of Hax-1 could restore the mitochondrial membrane potential and attenuate cell apoptosis. Moreover, pretreatment of MCAO mice with UCF-101 (7.15 mg/kg, ip) could restore Hax-1 expression, inhibit caspase activation, and significantly reduce the infarct volume.

Conclusion: Protease Omi impairs mitochondrial function by cleaving Hax-1, which induces apoptosis in OGD/R-treated N2a cells and causes I/R injury in MCAO mice.
\end{abstract}

Keywords: brain ischemia; MCAO mice; oxygen-glucose deprivation; neuroblastoma N2a cells; Omi; Hax-1; UCF-101; mitochondria; apoptosis

Acta Pharmacologica Sinica (2015) 36: 1043-1052; doi: 10.1038/aps.2015.50; published online 24 Aug 2015

\section{Introduction}

Ischemic stroke is one of the most common causes of longterm adult disability and the principal cause of death worldwide. It is known that global ischemia is caused by cardiac arrest, which leads to a reduced cerebral blood flow throughout the brain, whereas focal ischemia is caused by an embolic occlusion of the middle cerebral artery, resulting in a reduction of blood flow in a specific brain region ${ }^{[1]}$. Multiple events

\footnotetext{
* To whom correspondence should be addressed.

E-mail wanggh@suda.edu.cn (Guang-hui WANG); qshu@suda.edu.cn (Qing-song HU)

Received 2015-03-09 Accepted 2015-05-04
}

are known to be involved in neuronal death after ischemia, including excitotoxicity ${ }^{[2,3]}$, oxidative stress ${ }^{[4]}$, inflammation ${ }^{[5,6]}$, necrosis and apoptosis ${ }^{[7]}$. Apoptosis plays a critical role in ischemic brain injury ${ }^{[7]}$. As previously reported, apoptotic changes of neurons in the early stage of focal cerebral ischemia are presented in the ischemic core, including the condensation of nuclei and the activation of caspases, before they undergo necrosis ${ }^{[8]}$. In the ischemic penumbra, the rim of the ischemic core, neurons undergo both caspase-dependent and -independent apoptosis, which contributes to a delayed neuronal death $^{[9]}$.

Mitochondria are the cellular organelles that play critical roles in the apoptotic pathway in ischemic injury ${ }^{[10]}$. As previ- 
ously reported, cerebral ischemia/reperfusion (I/R) generates free radicals, mainly released by mitochondria, resulting in an oxidative stress in neurons ${ }^{[11]}$. Overproduction of reactive oxygen species (ROS) by mitochondria causes damage of proteins and lipids, which impairs mitochondrial function and leads to an increase of mitochondrial membrane permeability ${ }^{[12]}$. As a result, the permeabilized mitochondria release cytochrome $c$, activating caspases and leading to apoptotic cell death after $\mathrm{I} / \mathrm{R}^{[13]}$

The serine protease Omi/HtrA2 is a mammalian homolog of the bacterial heat-inducible protease HtrA/DegP and DegS ${ }^{[14]}$. Under physiological conditions, Omi is mainly localized in mitochondria and plays an important role in the maintenance of mitochondrial homeostasis ${ }^{[15]}$. Mutations that cause loss of Omi protease activity are usually associated with neurodegeneration such as Parkinson's disease ${ }^{[16]}$. Omi is a protease that is able to cleave its substrates involved in both normal function and disease ${ }^{[17]}$. Upon apoptotic stimuli, Omi is released from the mitochondria into the cytosol and cleaves its substrates, such as inhibitor of apoptosis proteins (IAPs), thereby inducing apoptosis ${ }^{[18]}$. In an ischemic brain, Omi is involved in neuronal injury after $I / R^{[19]}$. Once Omi is released from damaged mitochondria into the cytosol, the cytosolic substrates of Omi, including anti-apoptotic protein XIAP, are cleaved, thus causing apoptosis following focal cerebral I/ $\mathrm{R}^{[20]}$. Although the functions of Omi in apoptosis are extensively documented, the functions of Omi in mitochondria under physiological and pathological conditions are still unclear. Because Omi is a mitochondrial protein, the identification of its role in mitochondrial damage after I/R will help to provide an understanding of its function under pathological conditions. It was reported that an inhibition of Omi protease activity by UCF-101 shows protective effects on neurons after $I / \mathrm{R}^{[21]}$. Interestingly, in the penumbra after focal cerebral ischemia, an increase of Omi corresponds to a decrease of the mitochondrial protein Hax-1 (Hs1-associated protein X-1), a protein belonging to the Bcl-2 family ${ }^{[22]}$, suggesting a link between these two proteins in ischemic injury. However, the underlying mechanism of how Omi regulates Hax-1 after I/R is still largely unknown.

In this study, we show that Omi processes specificity for the mitochondrial anti-apoptotic protein Hax-1, thus causing mitochondrial damage and dysfunction and eventually stimulating cerebral ischemic neuronal cell death after I/R.

\section{Materials and methods Animal experiments}

Male ICR mice, 25-30 g, were purchased from SLACCAL Lab Animal Ltd (Shanghai, China). For the middle cerebral artery occlusion (MCAO) surgery ${ }^{[23]}$, mice were anesthetized with an intraperitoneal injection of $1 \%$ pentobarbital sodium. Then, the cerebral focal ischemia was produced by intra-luminal occlusion of the right MCA using a silicone coated nylon (6-0) monofilament (Doccol Corporation, Redlands, CA, USA). Two hours later, the occluding filament was withdrawn to allow blood reperfusion. Cerebral blood flow was monitored (LDF,
ML191 Laser Doppler Blood Flow Meter, Australia) and only those mice with a $90 \%$ of blood flow reduction during MCAO and a $85 \%-95 \%$ recovery of blood flow during reperfusion were used for further experiments. A homoeothermic heating blanket was used to maintain the core body temperature at $37^{\circ} \mathrm{C}$ during the $\mathrm{I} / \mathrm{R}$ operation. Mice in the sham group underwent identical operations except for the insertion of a filament. In the UCF-101 (Calbiochem, Darmstadt, Germany) treatment group, mice were given an intraperitoneal injection of vehicle or UCF-101 (7.15 mg/kg). Two hours after injection, the mice were subjected to MACO. All animals were used in accordance with the institutional guidelines for animal care and use outlined in a protocol that was approved by the Ethical Committee of Soochow University.

\section{Cell culture and oxygen glucose deprivation/reoxygenation (OGD/R) injury}

Mouse neuroblastoma (N2a) cells were maintained in Dulbecco's modified Eagle's medium (DMEM) containing 10\% fetal bovine serum with $100 \mu \mathrm{g} / \mathrm{mL}$ penicillin and $100 \mu \mathrm{g} / \mathrm{mL}$ streptomycin. To model OGD/R injury in N2a cells, the cells were incubated in glucose-free DMEM and saturated with $5 \% \mathrm{CO}_{2} / 95 \% \mathrm{~N}_{2}$ for $4 \mathrm{~h}$. After OGD exposure, the cells were subjected to reoxygenation with DMEM containing glucose in normoxia for $24 \mathrm{~h}$. For the MG132, bafilomycin A1 and UCF101 treatments, cells were treated with MG132 $(10 \mu \mathrm{mol} / \mathrm{L})$, bafilomycin A1 (1 $\mu \mathrm{mol} / \mathrm{L})$ or UCF-101 (10 $\mu \mathrm{mol} / \mathrm{L})$ for $12 \mathrm{~h}$ while being reoxygenated. Control cells were cultured in DMEM containing glucose in normoxia. Cells with or without treatment were subjected to immunofluorescent staining or immunoblot analyses.

\section{RNA extraction and real-time quantitative RT-PCR}

Total RNA was isolated from normoxia or OGD/R treated N2a cells with TRIzol reagent (Invitrogen, Carlsbad, CA, USA). cDNA was obtained using a reverse transcription reagent kit (Takara, Otsu, Shiga, Japan). Quantitative PCR (qPCR) was performed with Power SYBR Green PCR Master Mix (Applied Biosystems, Warrington, Cheshire, UK) and the products were detected using an Applied Biosystems 7500 Real Time PCR System. The sequences of qPCR primers used for mRNA quantification were as follows: 5'-AGCCCTGGCTATATCCGACA-3' and 5'-GAGGTCCAGGAAAGCCGAAA-3' for mouse Hax-1 and 5'-TGTGTCCGTCGTGGATCTGA-3' and 5'-TTGCTGTTGAAGTCGCAGGAG-3' for mouse GAPDH. Relative genomic expression was calculated by the $2^{-\Delta \Delta C t}$ method.

\section{Cell viability assay}

Cell viability was assessed with a MTT assay. N2a cells overexpressing Hax-1 or treated with UCF-101 were incubated with $0.5 \mathrm{mg} / \mathrm{mL} 3$-(4,5-dimethylthiazol-2-yl)-2,5-diphenyltetrazolium bromide (MTT) at $37^{\circ} \mathrm{C}$ for $2 \mathrm{~h}$. Then, $150 \mu \mathrm{L}$ of dimethylsulfoxide was added to stop the reaction. The absorbance was measured at $570 \mathrm{~nm}$ to determine cell viability. 


\section{Infarct volume measurement}

Brain infarct volume was determined $24 \mathrm{~h}$ after reperfusion. Mice were euthanized, and each brain was cut into five coronal slices (2-mm thick) with a brain-cutting matrix. The slices were stained with $1 \%$ TTC at $37^{\circ} \mathrm{C}$ for 30 min in the dark. Normal tissues were stained (red) and infarct tissues were not stained (white). The slices were mounted to dry paper and photographed with a scanner. The percentage of the infarct volume was quantified using Image-Proplus software, which calculated the ratio of infarct volumes to the total contralateral hemispheric volume. The ratios were multiplied by 100 .

\section{Plasmids}

The Hax-1 related constructs were generated as previously described ${ }^{[24]}$. Briefly, full length Hax-1 cDNA was amplified from a human fetal brain library (Invitrogen) with the primers 5'-GCGAATTCACCATGAGCCTCTTTGATCTCTTC-3' and 5'-ATGTCGACATCCGGGACCGGAACCAACGTCCC-3', and inserted into a pEGFP-N1 (Clontech, Mountain View, CA, USA) vector at the Hind III and Sal I sites.

\section{RNA interference}

The oligonucleotide target sequences to Omi (si-Omi) or the negative control siRNA (si-NC) have been previously described $^{[17,25]}$. The oligonucleotides were transfected with RNAiMAX (Invitrogen) following the manufacturer's instructions.

\section{Immunoblot analysis and antibodies}

Cells or tissue homogenates of ischemic or control brain hemispheres were lysed in $1 \times$ SDS lysis buffer $(25 \mathrm{mmol} / \mathrm{L}$ Tris$\mathrm{HCl}$, pH 7.6, $150 \mathrm{mmol} / \mathrm{L} \mathrm{NaCl}, 1 \% \mathrm{NP}-40$, and 1\% sodium deoxycholate) in the presence of a protease inhibitor cocktail (Roche, Mannheim, Germany). Approximately $20 \mu \mathrm{g}$ of cell lysate was separated by SDS-PAGE and transferred onto a PVDF membrane (Millipore, Billerica, MA, USA). Immunoblot analyses were performed with the following primary antibodies: anti-Hax-1 (Thermo Fisher Scientific, Waltham, MA, USA), anti-Omi (R\&D Systems, Minneapolis, MN, USA or prepared by our laboratory), anti-Tom20 (Santa Cruz Biotechnology, Santa Cruz, CA, USA), anti-GFP (Santa Cruz Biotechnology), anti-cleaved caspase-3 (Asp175, Cell Signaling Technology, Danvers, MA, USA). The secondary antibodies, sheep anti-mouse or anti-rabbit IgG-HRP, were from Thermo Fisher Scientific. The proteins were visualized using an ECL detection kit (Thermo Fisher Scientific).

\section{Immunofluorescence}

Cells were fixed with $4 \%$ paraformaldehyde in PBS for $5 \mathrm{~min}$ at room temperature, washed with PBS three times and permeabilized in $0.25 \%$ Triton X-100 in PBS for $3 \mathrm{~min}$. Thirty minutes after pre-blocking with $0.5 \%$ fetal bovine serum, cells were incubated with anti-Omi, anti-GFP or anti-Tom20 antibody for $6 \mathrm{~h}$, followed by an incubation with rhodamine (red)or FITC (green)-conjugated secondary antibody (Invitrogen, Carlsbad, CA, USA) for $2 \mathrm{~h}$. The cells were then stained with
DAPI (Sigma, St Louis, MO, USA) for 2 min. Finally, the cells were observed using an inverted IX71 microscope system (Olympus, Tokyo, Japan).

\section{Hoechst staining assays}

N2a cells overexpressing Hax-1 or treated with UCF-101 were incubated with Hoechst 33342 for 5 min and then washed with PBS. The cells were observed using an inverted IX71 microscope system (Olympus, Tokyo, Japan).

\section{Mitochondrial membrane potential measurement}

To measure the mitochondrial membrane potential $(\Delta \Psi \mathrm{m})$, cells were incubated with $100 \mathrm{nmol} / \mathrm{L}$ tetramethylrhodamine methyl ester (TMRM) in PBS for $15 \mathrm{~min}$ at $37^{\circ} \mathrm{C}$. TMRM is readily sequestered by normal mitochondria, but its fluorescence is rapidly lost when $\Delta \Psi \mathrm{m}$ is lost. After incubation, the cells were washed once with PBS and then resuspended in PBS. Finally, the cells were observed with an inverted IX71 microscope system (Olympus, Tokyo, Japan).

\section{Statistical analysis}

Densitometric analyses of immunoblots from three independent experiments were performed using Photoshop 7.0 (Adobe, San Jose, CA, USA). The data were analyzed using Origin 6.0 (Originlab, Northampton, MA, USA). Quantitative data are presented as the mean \pm SEM. Statistical significance was assessed via one-way ANOVA and the criterion of significance was set at $P<0.05$.

\section{Results}

\section{Cleavage of Hax-1 by Omi after ischemia/reperfusion}

Previous studies have shown that Omi levels increased and Hax-1 levels decreased in the penumbra after $I / R^{[22]}$. To explore how Omi regulates Hax-1 after I/R, we first examined the protein levels of Omi and Hax-1 in the penumbra of MCAO mouse brains $24 \mathrm{~h}$ after I/R. Consistent with the previous findings ${ }^{[22]}$, protein levels of Omi were increased (Figure 1A), while Hax-1 levels were decreased (Figure 1B). In addition, although Hax-1 protein levels were decreased in cells exposed to OGD/R (Figure 1C), transcription of Hax-1 was not altered (Figure 1D), suggesting a post-translational process of Hax-1. As we previously found that Omi cleaves Hax-1 ${ }^{[24]}$ and that Hax-1 is degraded by the ubiquitin-proteasome pathway after apoptotic stimulation ${ }^{[26]}$, we performed experiments using a cellular OGD/R model to address how Omi regulates Hax-1 after I/R. In N2a cells that were exposed to OGD/R, an increase of Omi and a decrease of Hax-1 were observed, however, Hax-1 protein levels were dramatically increased after Omi was knocked down (Figure 1E), suggesting that Omi possibly processes Hax-1. We further examined the Hax-1 levels in OGD/R cells with or without the administration of the proteasomal inhibitor MG132 or the lysosomal inhibitor bafilomycin A1 (BafA1) to differentiate Hax-1 degradation pathways. MG132 or BafA1 did not block the degradation of Hax-1 (Figure $1 \mathrm{~F}$ ), further supporting that Hax-1 is processed by Omi but not degraded by the proteasomal or lysosomal pathway. 
Involvement of Hax-1 in the protective effects against OGD/ R-induced neuronal injury

As Hax-1 is an anti-apoptotic protein belonging to Bcl-2 family, we questioned whether a decrease of Hax-1 level is associated with cell death induced by OGD/R. Therefore, we overexpressed EGFP alone or EGFP-tagged Hax-1 and evaluated cell viability after OGD/R. Significant cell death was observed in EGFP alone transfected cells after OGD/R; however, overexpression of EGFP-Hax-1 significantly blocked OGD/R-induced cell death (Figure 2A).

We have shown that there is a correlation between Omi and Hax-1 levels (Figure 1). We questioned whether OGD/R-induced cell death mediated by the regulation of Hax-1 is associated with the increase of Omi and Omi protease
A
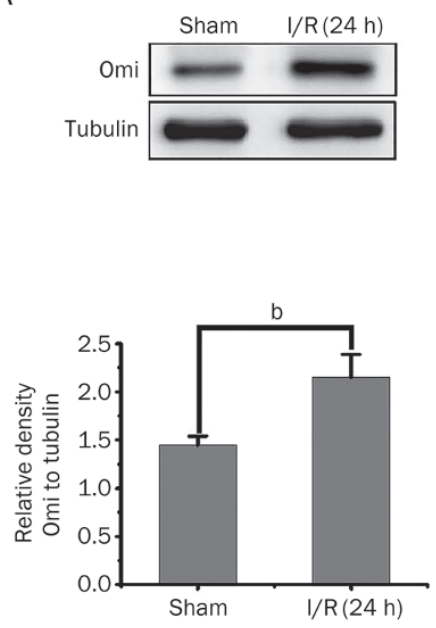

B
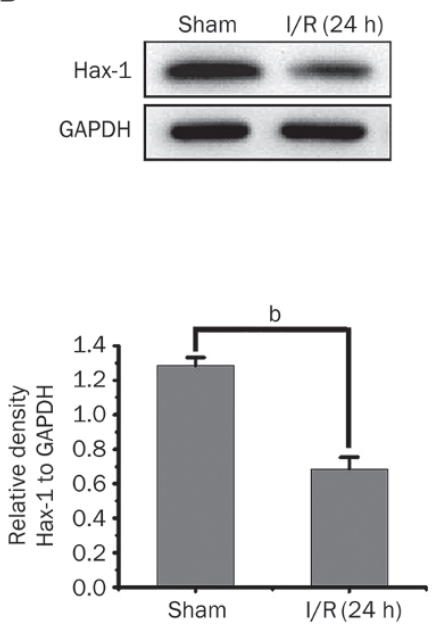

C
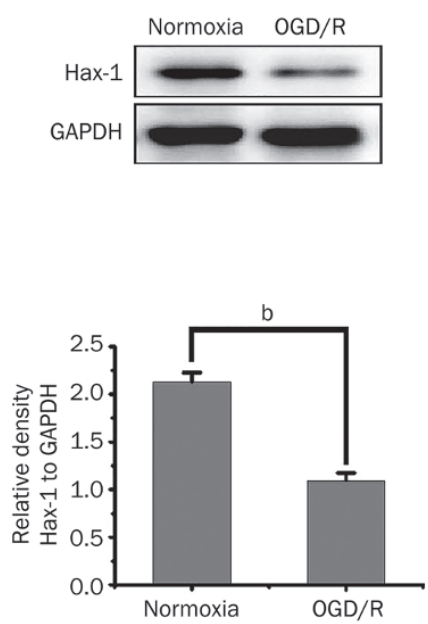

D

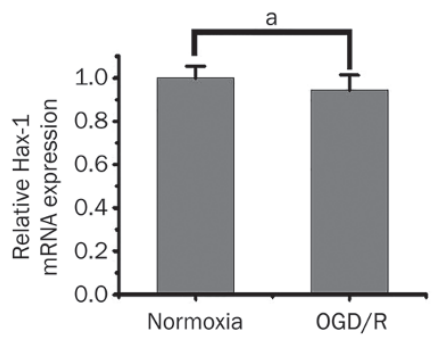

E
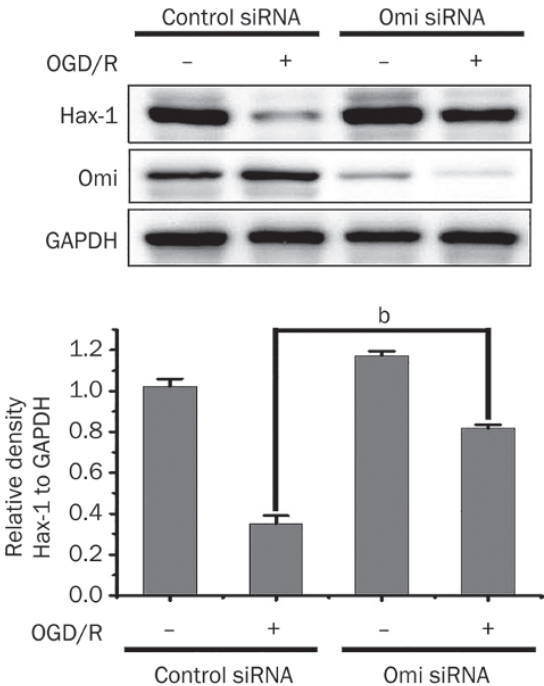

$\mathrm{F}$
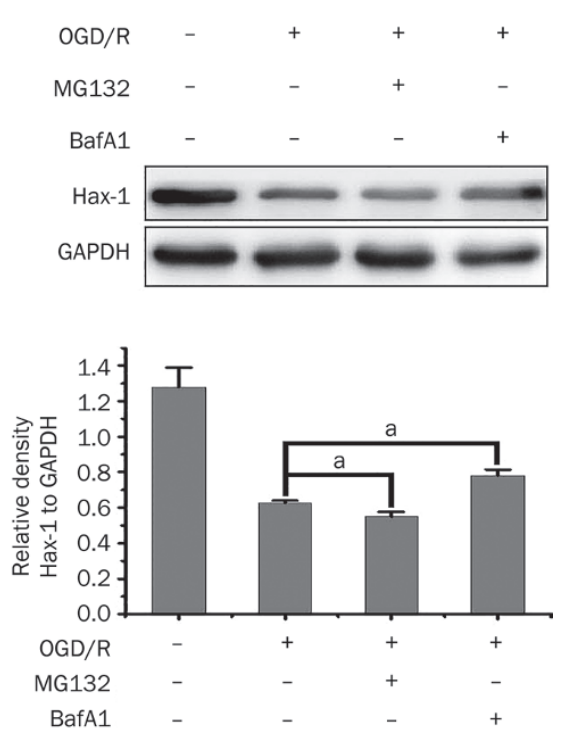

Figure 1. Omi processes Hax-1 after $\mathrm{I} / \mathrm{R}$ in vivo and OGD/R in vitro. (A and B) Immunoblot analyses were performed to show Omi (A) and Hax-1 (B) protein levels in the penumbra after I/R. ICR mice $(n=3)$ were subjected to a middle cerebral artery occlusion $(\mathrm{MCAO})$ for $2 \mathrm{~h}$, followed by $24 \mathrm{~h}$ of reperfusion. The bottom panels show the band intensity of Omi or Hax-1 relative to that of tubulin (A) or GAPDH (B), respectively. (C) Immunoblot analyses were performed to show Hax-1 protein levels in N2a cells that were exposed to OGD for $4 \mathrm{~h}$, followed by $12 \mathrm{~h}$ of reoxygenation. The bottom panel shows the band intensity of Hax-1 relative to that of GAPDH. (D) Real-time quantitative PCR was performed to examine Hax-1 mRNA levels in N2a cells that were subjected to OGD for $4 \mathrm{~h}$, followed by $12 \mathrm{~h}$ of reoxygenation. The Hax-1 mRNA levels were quantified and normalized to GAPDH. (E) Immunoblot analyses were performed to show Hax-1 levels in OGD/R N2a cells in which Omi was knocked down. N2a cells were subjected to OGD for $4 \mathrm{~h}$, followed by $12 \mathrm{~h}$ of reoxygenation. The bottom panel shows the band intensity of Hax-1 relative to that of GAPDH. (F) Immunoblot analyses were performed to show Hax-1 levels in OGD/R N2a cells that were treated with MG132 $(10 \mu \mathrm{mol} / \mathrm{L})$ and bafilomycin A1 $(1 \mu \mathrm{mol} / \mathrm{L})$. The bottom panel shows the band intensity of Hax-1 relative to that of GAPDH. Values are the mean \pm SEM from three independent experiments. ${ }^{a} P>0.05,{ }^{b} P<0.05$, one-way ANOVA. 
activity. We treated the OGD/R cells with UCF-101, an Omi protease inhibitor, and examined cell viability after OGD/R. In cells exposed to OGD/R, there was a decrease of cell viability and Hax-1 protein levels along with an increase of Omi levels; however, inhibition of Omi protease activity by UCF101 greatly restored cell viability and Hax-1 levels after OGD/R (Figure 2B).

\section{Maintenance of mitochondrial integrity by Hax-1 in OGD/R cells}

Mitochondria are important cellular organelles that are involved in neuronal cell death after I/ $\mathrm{R}$ by releasing cytochrome $c^{[13]}$. Because Hax-1 is decreased in OGD/R cells as well as in the brains after I/R (Figure 1), we questioned whether the increase of Omi and the decrease of Hax-1 are associated with mitochondrial damage in OGD/R cells. Omi and Hax-1 both localize to mitochondria, as shown by colocalization with mitochondrial protein Tom20 (Figure 3A and $3 \mathrm{~B})$. In normoxic cells, inhibition of Omi protease activ- ity by UCF-101 did not affect the mitochondrial membrane potential (Figure 3C). In OGD/R cells, there was a significant decrease of mitochondrial membrane potential, indicated by TMRM staining (Figure 3C). However, administration of UCF-101 significantly restored the mitochondrial membrane potential (Figure 3C). Moreover, overexpression of Hax-1 also restored the mitochondrial membrane potential in OGD/R cells, showing a similar effect as UCF-101 treatment (Figure 3D). These data suggest that an inhibition of Omi protease activity or overexpression of Hax-1 is able to maintain mitochondrial integrity against OGD/R injury. Next, we examined whether an inhibition of Omi protease activity or overexpression of Hax-1 protects OGD/R cells against apoptosis. After OGD/R, a majority of the cells presented condensed nuclei (Figure $4 \mathrm{~A}$ and $4 \mathrm{~B}$ ), whereas, only a small portion of OGD/R cells presented apoptotic features after administration of UCF-101 (Figure 4A). Additionally, overexpression of Hax-1 significantly alleviated apoptotic changes induced by OGD/R
A
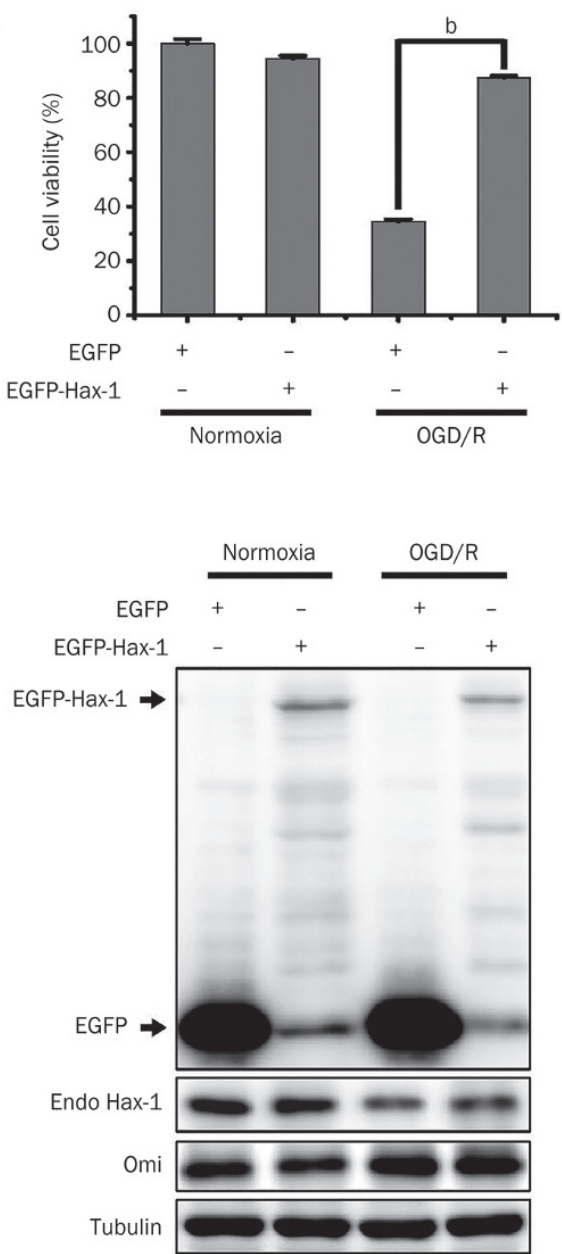
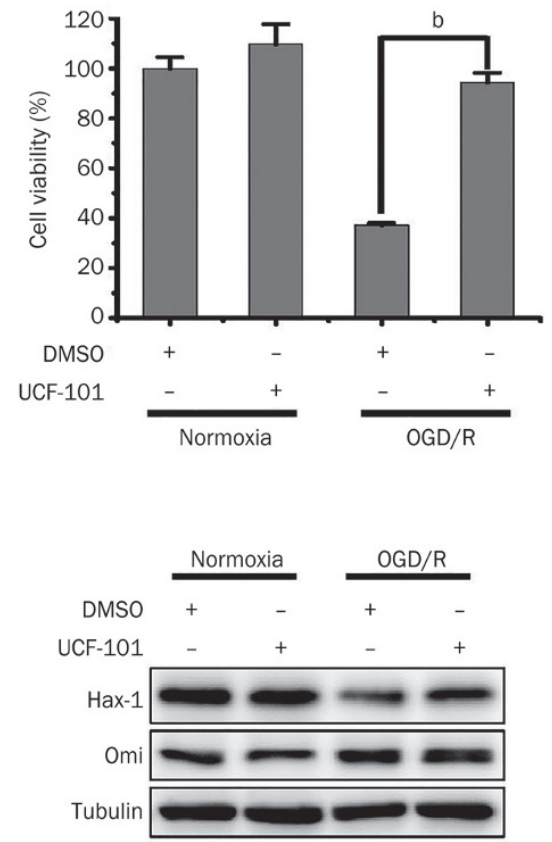

Figure 2. Inhibition of Omi protease activity or overexpression of Hax-1 protects N2a cells against OGD/R-induced cell death. (A) MTT assays were performed to show N2a cell viability after OGD/R. N2a cells were transfected with EGFP or EGFP-Hax-1 for $48 \mathrm{~h}$ and then subjected to OGD/R, followed by $12 \mathrm{~h}$ of reoxygenation. Immunoblot analyses were performed to show the levels of Hax-1 and Omi. (B) MTT assays were performed to show N2a cell viability after OGD/R and UCF-101 treatment. The N2a cells were subjected to OGD for $4 \mathrm{~h}$ and then treated with UCF-101 (10 $\mu \mathrm{mol} / \mathrm{L}$ ), followed by reoxygenation for $12 \mathrm{~h}$. Immunoblot analysis was performed to show the levels of Hax-1 and Omi. Values are the mean \pm SEM from three independent experiments. ${ }^{\mathrm{b}} \mathrm{P}<0.05$, one-way ANOVA. 
A

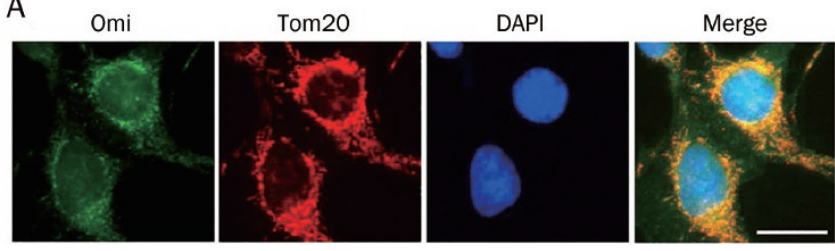

C

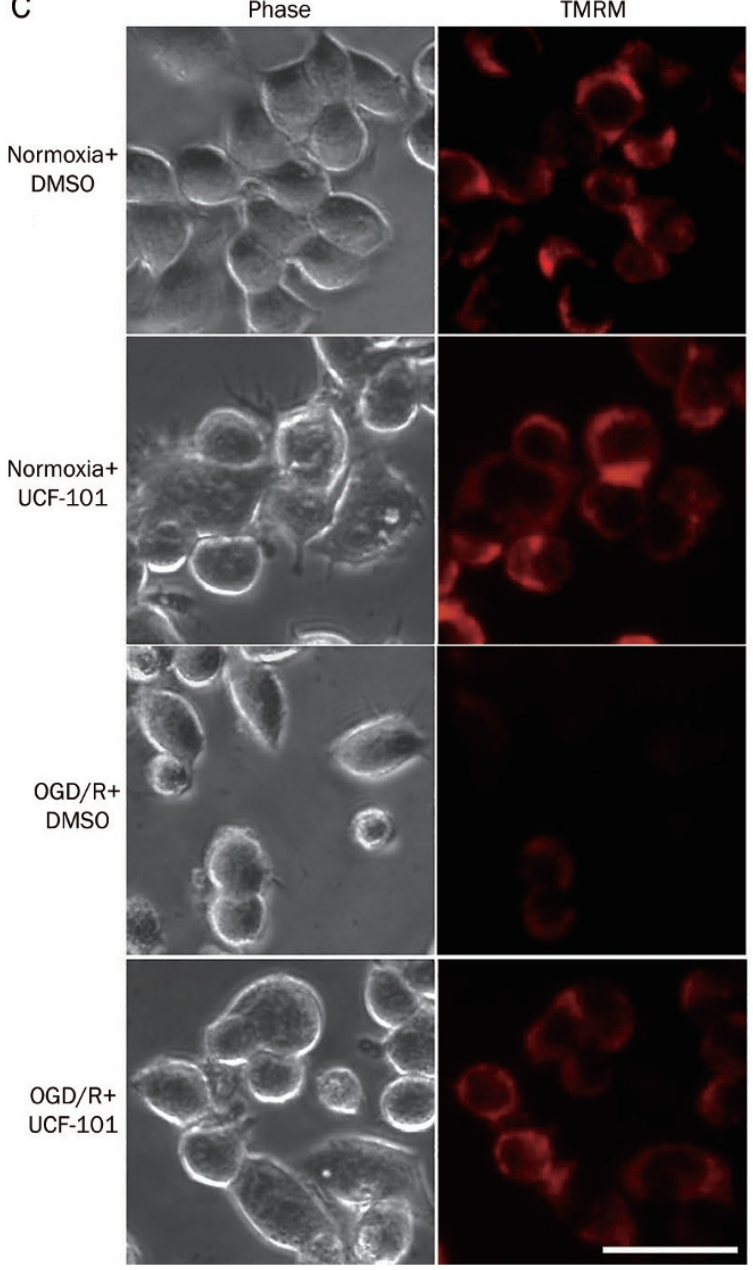

B

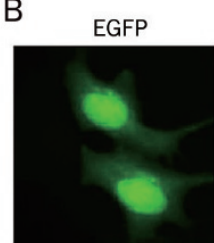

Tom20

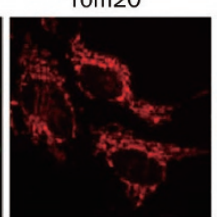

DAPI
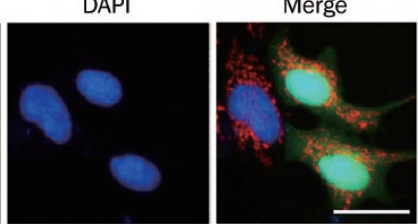

EGFP-Hax-1

Tom20
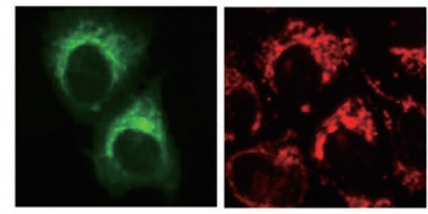

DAPI

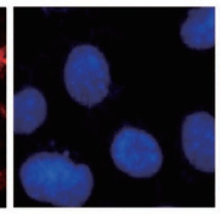

Merge

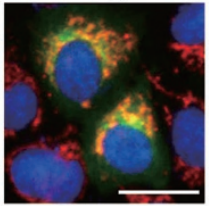

D

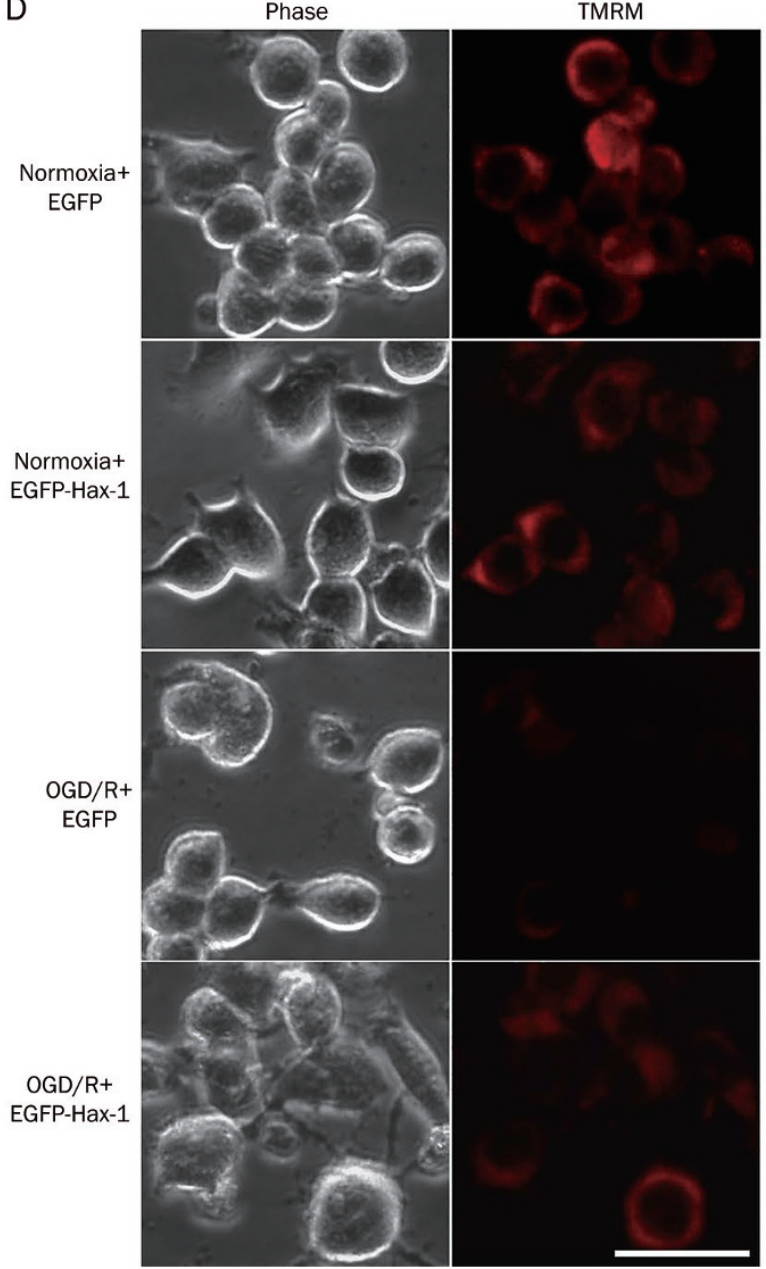

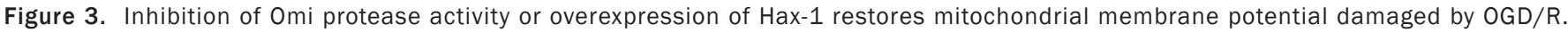

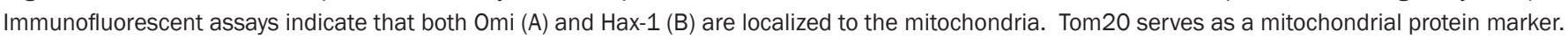

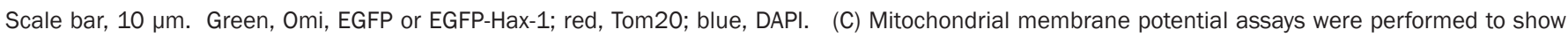

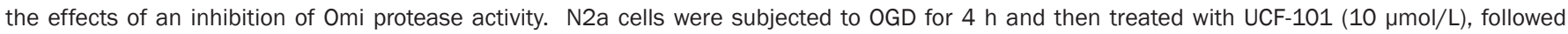

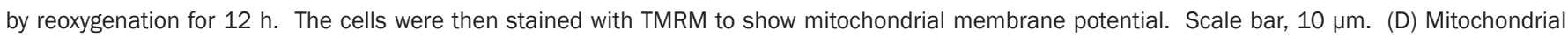

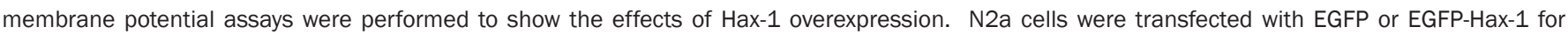

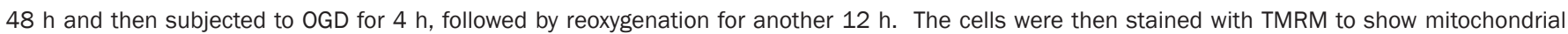
membrane potential. Scale bar, $10 \mu \mathrm{m}$. 
(Figure 4B). Consistent with the morphological data (Figure 4A and 4B), administration of UCF-101 (Figure 4C, left panel) or overexpression of Hax-1 (Figure 4C, right panel) in OGD/R cells decreased the levels of cleaved caspase-3. These data further suggest that Hax-1 protects OGD/R cells against apoptosis.

A

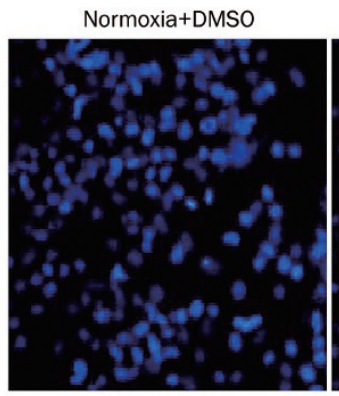

Normoxia+UCF-101

$\mathrm{OGD} / \mathrm{R}+\mathrm{DMSO}$
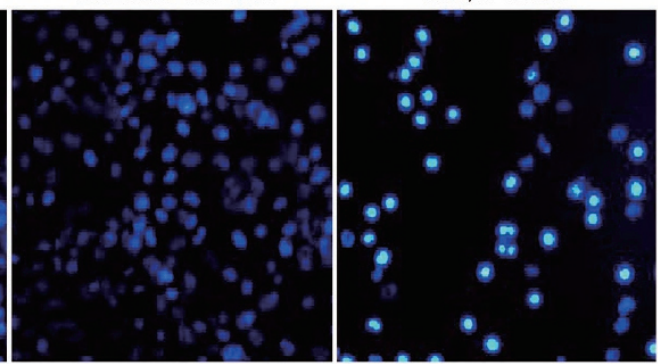

OGD/R+UCF-101

B
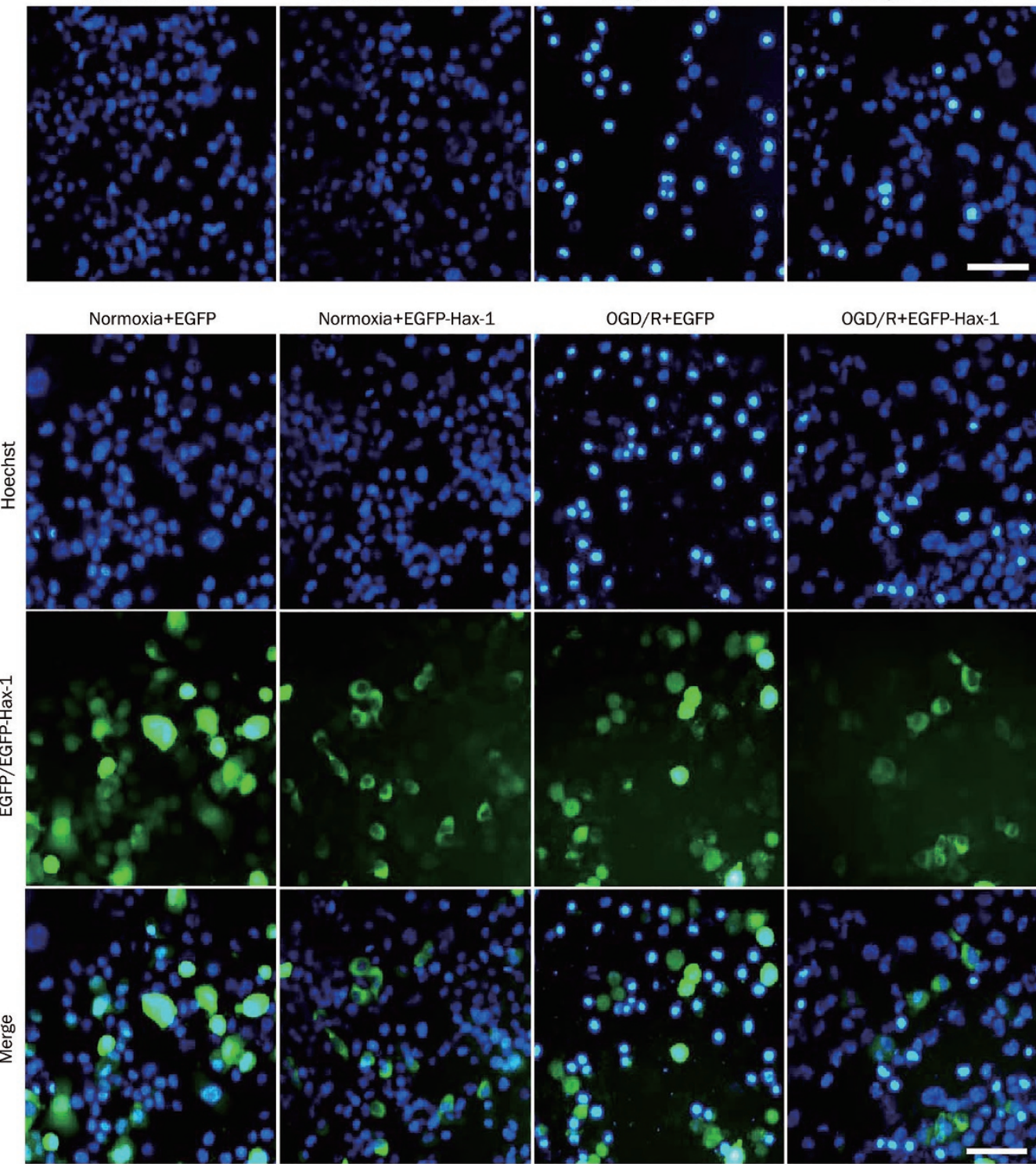

\section{OGD/R+EGFP \\ T}

OGD/R+EGFP-Hax-1
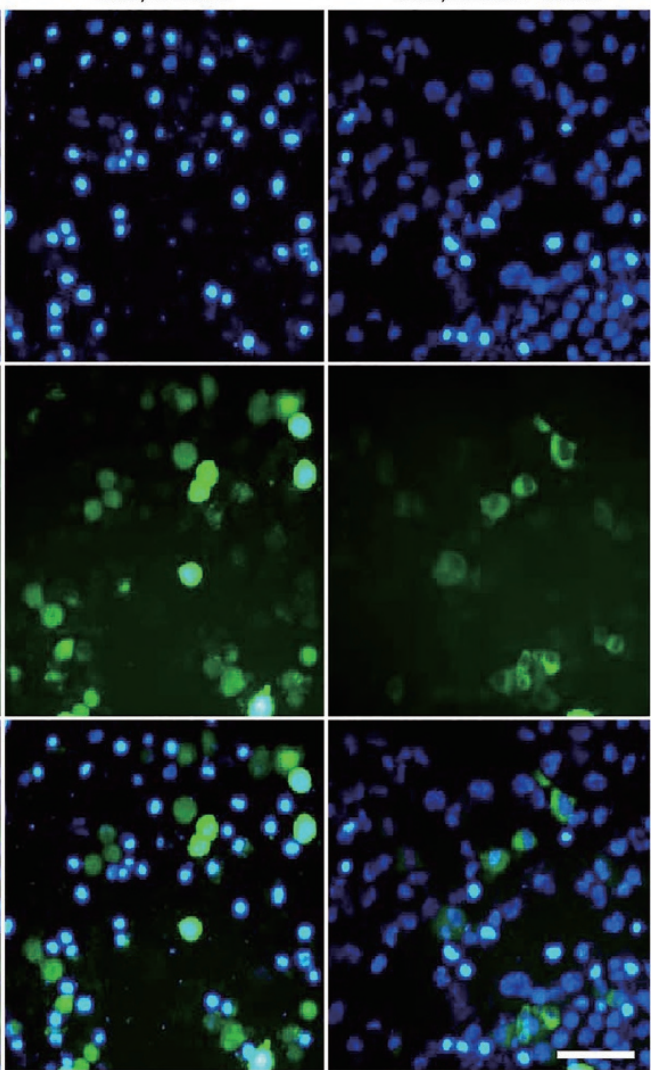

C
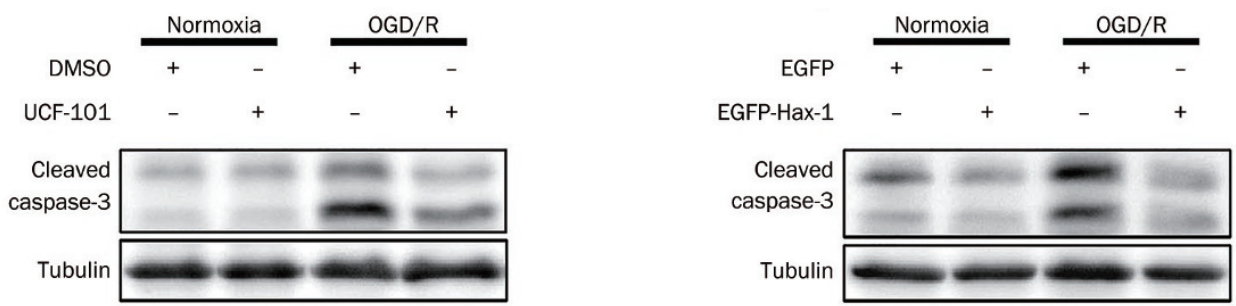

Figure 4. Inhibition of Omi protease activity or overexpression of Hax-1 protects OGD/R cells against apoptosis. (A) Hoechst staining was performed to show the effect of inhibition of Omi protease activity on apoptosis after OGD/R. N2a cells were subjected to OGD/R as in Figure 3C. Scale bar, $10 \mu m$. (B) EGFP/Hoechst double staining was performed to show the effects of Hax-1 overexpression on apoptosis after OGD/R. N2a cells were subjected to $\mathrm{OGD} / \mathrm{R}$ as in Figure 3D. Scale bar, $10 \mu \mathrm{m}$. (C) Immunoblot analyses were performed to show that inhibition of Omi protease activity or overexpression of Hax-1 decreases the level of cleaved caspase-3 in OGD/R cells. 


\section{Protective effects in MCAO mice by UCF-101}

We have shown that the anti-apoptotic effects on OGD/R cells were caused by an inhibition of Omi protease activity or overexpression of Hax-1 (Figure 3 and 4). Next, we questioned whether inhibition of Omi could protect neurons against apoptosis and neurons in MCAO mice to survive. To answer it, we examined Hax-1 expression in the penumbra after I/R. As shown in Figure 5A, Hax-1 levels in the penumbra were dramatically decreased after $I / R$, but were significantly restored by treatment with UCF-101. We then examined the cleavage of caspase-3. The cleavage of caspase- 3 was increased in the penumbra after I/R but was blocked after an administration of UCF-101 (Figure 5B). Thus, these data strongly suggest that UCF-101 inhibits Omi activity to decrease the processing of Hax-1, leading to an inhibition of caspase-3 activation in MCAO animals. Finally, we examined whether inhibition of Omi had an effect on neuronal survival. Using TTC staining, we evaluated the infarct volume of MCAO mice treated with vehicle or UCF-101 $24 \mathrm{~h}$ after I/R. In comparison to the sham group, MCAO mice with vehicle treatment presented an average infarct volume of approximately $27 \%(27.22 \% \pm 0.83 \%)$; however, the average infarct volume in MCAO mice treated with UCF-101 decreased to approximately 17\% (17.31\% $\pm 1.44 \%)$
(Figure 5C), suggesting a protective effect of UCF-101.

\section{Discussion}

Although multiple factors are involved in neuronal cell death after I/R, mitochondria-mediated apoptosis is one of the intrinsic pathways critical for ischemic injury ${ }^{[27]}$. Mitochondria are cellular organelles essential for many functions including oxidative phosphorylation, calcium homeostasis, ROS management and apoptosis ${ }^{[28]}$. Depletion of energy stores by I/ R leads to a loss of membrane potential and a depolarization of neurons, which activate $\mathrm{Ca}^{2+}$ channels, resulting in an elevation of free cytosolic $\mathrm{Ca}^{2+[29]}$. The depletion of energy and elevation of $\mathrm{Ca}^{2+}$ promote the production of ROS, further impairing mitochondria after $I / \mathrm{R}^{[30]}$. In addition, increased $\mathrm{Ca}^{2+}$ stimulates calpain to cleave Bid to form $\mathrm{tBid}$ that binds to pro-apoptotic proteins such as Bax, Bad, and Bak after I/ $\mathrm{R}^{[31]}$. The formation of $\mathrm{tBid}$ heterodimers with other pro-apoptotic protein opens the mitochondrial permeability transition pore (mPTP) and releases cytochrome $c$, thereby activating caspases, leading to apoptosis in the ischemic brain ${ }^{[32,33]}$.

The Bcl-2 protein family includes both anti-apoptotic (such as Bcl-2 and Bcl-xL) and pro-apoptotic proteins (such as Bax, Bad and Bak). Bcl-2 and Bcl-xL can form heterodimers with
A

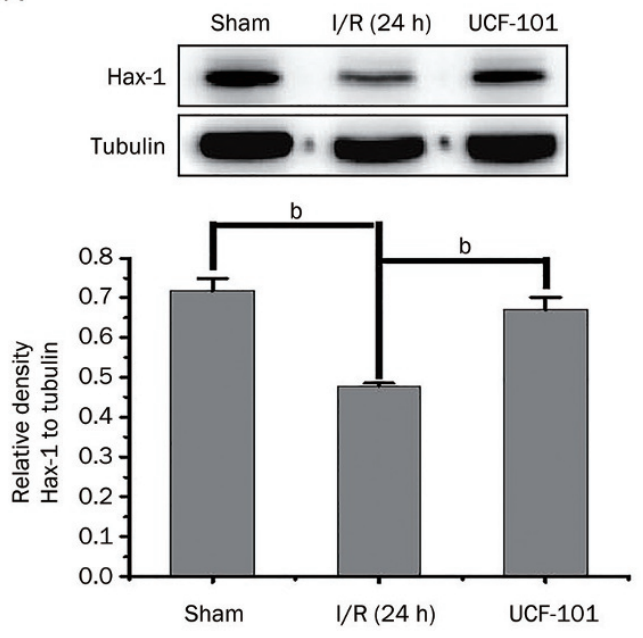

B

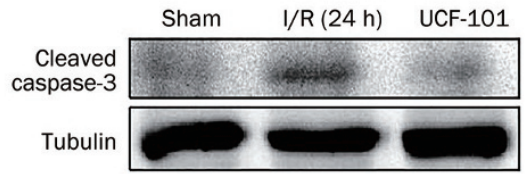

C
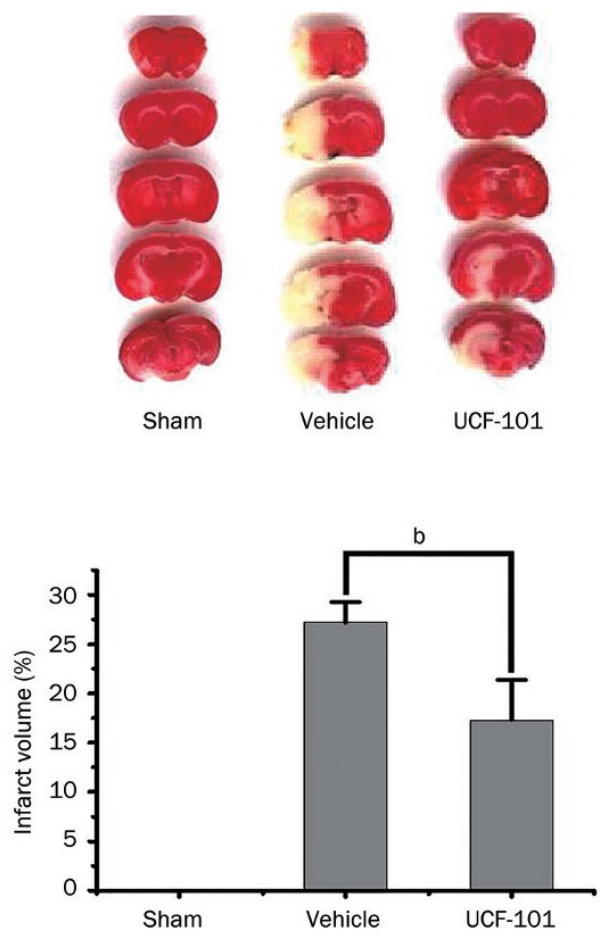

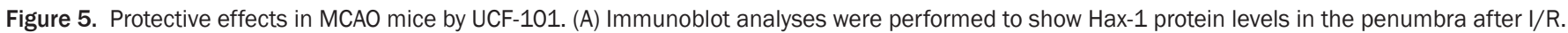

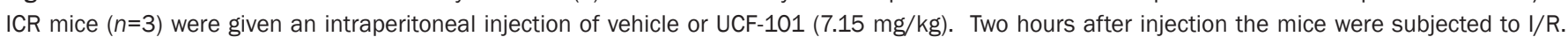

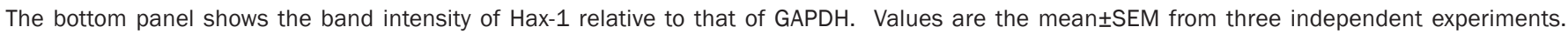

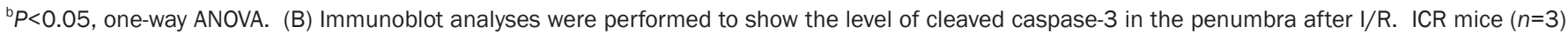

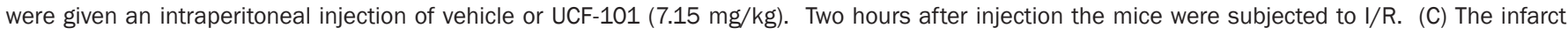

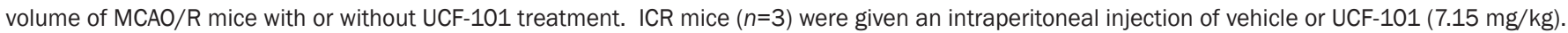

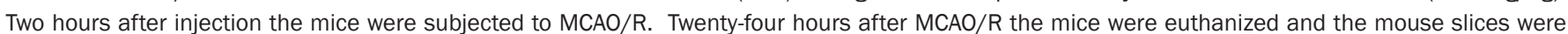
stained with TTC. The bottom panel shows the infarct volumes. ${ }^{\mathrm{b}} P<0.05$, one-way ANOVA. 
pro-apoptotic protein to protect mitochondria against apoptotic stimulation by ischemia ${ }^{[7,34]}$. Overexpression of Bcl- $2^{[35]}$ or systemic delivery of Bcl-xL ${ }^{[36]}$ blocks apoptosis inducing factor (AIF) translocation, prevents caspase activation and protects neurons after ischemia. Hax-1 is a mitochondrial protein belonging to Bcl-2 family. It was reported that Hax-1 interacts with caspase-9 to repress caspase-9 activation in myocytes ${ }^{[37]}$, suggesting an anti-apoptotic effect. Importantly, Hax-1 is critical for the regulation of mitochondrial membrane potential during apoptosis ${ }^{[38,39]}$. Peripheral blood neutrophils derived from Hax-1-deficient patients displayed an abnormal mitochondrial membrane potential and stem cells from these patients showed a rapid loss of mitochondrial membrane potential during apoptosis ${ }^{[40]}$. Most interestingly, restoration of Hax-1 levels in myeloid progenitor cells from Hax-1-deficient patients by retroviral gene manipulation delays the loss of mitochondrial membrane potential, making them have similar properties as the controls ${ }^{[40]}$. These studies suggest a role of Hax-1 in the regulation of mitochondrial membrane potential. In our study, we found that Hax-1 is processed by Omi after OGD/R or I/R. N2a cells after OGD/R showed apoptotic changes with a loss of mitochondrial membrane potential. Inhibition of Omi activity or an overexpression of Hax-1 restored mitochondrial membrane potential and protected cells from apoptosis. Moreover, inhibition of Omi's protease activity in MCAO mice increased Hax-1 levels and decreases the infarct volume. Thus, our data suggest that Hax-1 levels are critical for maintaining mitochondrial membrane potential to protect neurons against apoptosis after I/R.

The levels of Omi and Hax-1 are inversely altered after $\mathrm{I} / \mathrm{R}^{[22]}$ and both are localized to mitochondria. In our study, we demonstrate that Omi processes Hax-1 after OGD/R or $\mathrm{I} / \mathrm{R}$ to induce apoptosis. Blocking Omi enzyme activity, similar to Hax-1 overexpression, significantly inhibits caspase-3 and protects neurons against OGD/R or I/R-induced injury. Thus, we propose that after I/R, Omi first affects proteins on mitochondria before it is released to cytosol to cleave cytosolic substrates.

In summary, our findings reveal a role of Omi in ischemia/ reperfusion-induced apoptosis by its functioning on mitochondria to process the mitochondrial anti-apoptotic protein Hax-1.

\section{Acknowledgements}

This work was supported in part by the National Natural Science Foundation of China (No 31330030 and 31300887), the National High-tech Research and Development Program of China 973-projects (2012CB947602), Natural Science Foundation of Jiangsu Province (BK20130299), and a project funded by the Priority Academic Program Development of Jiangsu Higher Education Institutions.

\section{Author contribution}

Qing-song HU and Guang-hui WANG designed the experiments; Jia-yuan WU and Qing-song HU performed main parts of the experiments; Mei LI, Li-juan CAO, Dong CHEN, Haigang REN, Qin XIA, Zhou-teng TAO and Mei-ling SUN per- formed parts of the experiments; Qing-song HU, Zheng-hong QIN, and Guang-hui WANG analyzed the data; Qing-song $\mathrm{HU}$ and Guang-hui WANG wrote the manuscript.

\section{References}

1 Krafft PR, Bailey EL, Lekic T, Rolland WB, Altay O, Tang J, et al. Etiology of stroke and choice of models. Int J Stroke 2012; 7: 398406.

2 Connolly NM, Dussmann H, Anilkumar U, Huber HJ, Prehn JH. Singlecell imaging of bioenergetic responses to neuronal excitotoxicity and oxygen and glucose deprivation. J Neurosci 2014; 34: 10192-205.

3 Liu XQ, Sheng R, Qin ZH. The neuroprotective mechanism of brain ischemic preconditioning. Acta Pharmacol Sin 2009; 30: 1071-80.

4 Li L, Zhu K, Liu Y, Wu X, Wu J, Zhao Y, et al. Targeting thioredoxin-1 with siRNA exacerbates oxidative stress injury after cerebral ischemia/reperfusion in rats. Neuroscience 2015; 284: 815-23.

5 Benakis C, Garcia-Bonilla L, ladecola C, Anrather J. The role of microglia and myeloid immune cells in acute cerebral ischemia. Front Cell Neurosci 2014; 8: 461.

6 Clausen B, Degn M, Martin N, Couch Y, Karimi L, Ormhoj M, et al. Systemically administered anti-TNF therapy ameliorates functional outcomes after focal cerebral ischemia. J Neuroinflammation 2014; 11: 203.

7 Anilkumar U, Prehn JH. Anti-apoptotic BCL-2 family proteins in acute neural injury. Front Cell Neurosci 2014; 8: 281.

8 Benchoua A, Guegan C, Couriaud C, Hosseini H, Sampaio N, Morin D, et al. Specific caspase pathways are activated in the two stages of cerebral infarction. J Neurosci 2001; 21: 7127-34.

9 Ferrer I. Apoptosis: future targets for neuroprotective strategies. Cerebrovasc Dis 2006; 21 Suppl 2: 9-20.

10 Zuo W, Zhang S, Xia CY, Guo XF, He WB, Chen NH. Mitochondria autophagy is induced after hypoxic/ischemic stress in a Drp1 dependent manner: the role of inhibition of Drp1 in ischemic brain damage. Neuropharmacology 2014; 86: 103-15.

11 Nita DA, Nita V, Spulber S, Moldovan M, Popa DP, Zagrean AM, et al. Oxidative damage following cerebral ischemia depends on reperfusion - a biochemical study in rat. J Cell Mol Med 2001; 5: 163-70.

12 Yuan J, Yankner BA. Apoptosis in the nervous system. Nature 2000; 407: 802-9.

13 Cao G, Minami M, Pei W, Yan C, Chen D, O'Horo C, et al. Intracellular Bax translocation after transient cerebral ischemia: implications for a role of the mitochondrial apoptotic signaling pathway in ischemic neuronal death. J Cereb Blood Flow Metab 2001; 21: 321-33.

14 Vande Walle L, Lamkanfi M, Vandenabeele P. The mitochondrial serine protease HtrA2/Omi: an overview. Cell Death Differ 2008; 15: 453-60.

15 Radke S, Chander H, Schafer P, Meiss G, Kruger R, Schulz JB, et al. Mitochondrial protein quality control by the proteasome involves ubiquitination and the protease Omi. J Biol Chem 2008; 283: 12681-5.

16 Strauss KM, Martins LM, Plun-Favreau H, Marx FP, Kautzmann S, Berg $\mathrm{D}$, et al. Loss of function mutations in the gene encoding Omi/HtrA2 in Parkinson's disease. Hum Mol Genet 2005; 14: 2099-111.

$17 \mathrm{Hu}$ Q, Li B, Xu R, Chen D, Mu C, Fei E, et al. The protease Omi cleaves the mitogen-activated protein kinase kinase MEK1 to inhibit microglial activation. Sci Signal 2012; 5: ra61.

18 Suzuki Y, Imai Y, Nakayama H, Takahashi K, Takio K, Takahashi R. A serine protease, HtrA2, is released from the mitochondria and interacts with XIAP, inducing cell death. Mol Cell 2001; 8: 613-21.

19 Yoshioka H, Katsu M, Sakata H, Okami N, Wakai T, Kinouchi H, et al. 
The role of PARL and HtrA2 in striatal neuronal injury after transient global cerebral ischemia. J Cereb Blood Flow Metab 2013; 33 : 1658-65.

20 Althaus J, Siegelin MD, Dehghani F, Cilenti L, Zervos AS, Rami A. The serine protease Omi/HtrA2 is involved in XIAP cleavage and in neuronal cell death following focal cerebral ischemia/reperfusion. Neurochem Int 2007; 50: 172-80.

21 Su D, Su Z, Wang J, Yang S, Ma J. UCF-101, a novel Omi/HtrA2 inhibitor, protects against cerebral ischemia/reperfusion injury in rats. Anat Rec (Hoboken) 2009; 292: 854-61.

22 Rami A, Langhagen A. Specific alterations of the HtrA2/HAX-1 ratio in the penumbra upon focal cerebral ischemia in mice. Neurochem Res 2012; 37: 548-56.

23 Sheng R, Zhang LS, Han R, Gao B, Liu XQ, Qin ZH. Combined prostaglandin $\mathrm{E} 1$ and lithium exert potent neuroprotection in a rat model of cerebral ischemia. Acta Pharmacol Sin 2011; 32: 303-10.

24 Li B, Hu Q, Wang H, Man N, Ren H, Wen L, et al. Omi/HtrA2 is a positive regulator of autophagy that facilitates the degradation of mutant proteins involved in neurodegenerative diseases. Cell Death Differ 2010; 17: 1773-84.

25 Ma Q, Hu QS, Xu RJ, Zhen XC, Wang GH. Protease omi facilitates neurite outgrowth in mouse neuroblastoma N2a cells by cleaving transcription factor E2F1. Acta Pharmacol Sin 2015; 36: 966-75.

26 Li B, Hu Q, Xu R, Ren H, Fei E, Chen D, et al. Hax-1 is rapidly degraded by the proteasome dependent on its PEST sequence. BMC Cell Biol 2012; 13: 20.

27 Nakka VP, Gusain A, Mehta SL, Raghubir R. Molecular mechanisms of apoptosis in cerebral ischemia: multiple neuroprotective opportunities. Mol Neurobiol 2008; 37: 7-38.

28 Tait SW, Green DR. Mitochondria and cell signalling. J Cell Sci 2012; 125: 807-15.

29 Lo EH, Dalkara T, Moskowitz MA. Mechanisms, challenges and opportunities in stroke. Nat Rev Neurosci 2003; 4: 399-415.

30 Mergenthaler P, Dirnagl U, Meisel A. Pathophysiology of stroke: lessons from animal models. Metab Brain Dis 2004; 19: 151-67.

31 Plesnila N, Zinkel S, Amin-Hanjani S, Qiu J, Korsmeyer SJ, Moskowitz MA. Function of BID - a molecule of the bcl-2 family - in ischemic cell death in the brain. Eur Surg Res 2002; 34: 37-41.

32 Fan YY, Shen Z, He P, Jiang L, Hou WW, Shen Y, et al. A novel neuroprotective strategy for ischemic stroke: transient mild acidosis treatment by $\mathrm{CO}_{2}$ inhalation at reperfusion. J Cereb Blood Flow Metab 2014; 34: 275-83.

33 Saito A, Hayashi T, Okuno S, Ferrand-Drake M, Chan PH. Overexpression of copper/zinc superoxide dismutase in transgenic mice protects against neuronal cell death after transient focal ischemia by blocking activation of the Bad cell death signaling pathway. J Neurosci 2003; 23: 1710-8.

34 Gibson ME, Han BH, Choi J, Knudson CM, Korsmeyer SJ, Parsadanian $\mathrm{M}$, et al. BAX contributes to apoptotic-like death following neonatal hypoxia-ischemia: evidence for distinct apoptosis pathways. Mol Med 2001; 7: 644-55.

35 Zhao H, Yenari MA, Cheng D, Barreto-Chang OL, Sapolsky RM, Steinberg GK. Bcl-2 transfection via herpes simplex virus blocks apoptosis-inducing factor translocation after focal ischemia in the rat. J Cereb Blood Flow Metab 2004; 24: 681-92.

36 Yin W, Cao G, Johnnides MJ, Signore AP, Luo Y, Hickey RW, et al. TAT-mediated delivery of $\mathrm{Bcl}-\mathrm{xL}$ protein is neuroprotective against neonatal hypoxic-ischemic brain injury via inhibition of caspases and AlF. Neurobiol Dis 2006; 21: 358-71.

37 Han Y, Chen YS, Liu Z, Bodyak N, Rigor D, Bisping E, et al. Overexpression of HAX-1 protects cardiac myocytes from apoptosis through caspase-9 inhibition. Circ Res 2006; 99: 415-23.

38 Cilenti L, Soundarapandian MM, Kyriazis GA, Stratico V, Singh S, Gupta S, et al. Regulation of HAX-1 anti-apoptotic protein by Omi/ HtrA2 protease during cell death. J Biol Chem 2004; 279: 50295301.

39 Sharp TV, Wang HW, Koumi A, Hollyman D, Endo Y, Ye H, et al. K15 protein of Kaposi's sarcoma-associated herpesvirus is latently expressed and binds to HAX-1, a protein with antiapoptotic function. J Virol 2002; 76: 802-16.

40 Klein C, Grudzien M, Appaswamy G, Germeshausen M, Sandrock I, Schaffer AA, et al. HAX1 deficiency causes autosomal recessive severe congenital neutropenia (Kostmann disease). Nat Genet 2007; 39: 86-92. 